Vol. 1

No. 1

Jul - Dic 2016

ISSN: $2590-9215$

(En línea)

PP: $38-49$

\title{
Factores Incidentes en la Participación de la Mujer en el Mercado Laboral Hondureño, 2013*
}

\author{
Incident Factors in Women's Participation in Honduran Labor Market, 2013 \\ Fatores Incidentes na participação das mulheres no Mercado de \\ Trabalho na Honduras, 2013*.
}

\author{
Kenia Maryorie Meza-Hernández ${ }^{* *}$, José Bayardo Cabrera-Rosales***, \\ Mario Josué Ramos-Canales ${ }^{* * * *}$
}

Forma de citar: Meza K. M., Cabrera, J. B., y Ramos, M. J. (2016). Factores Incidentes en la Participación de la Mujer en el Mercado Laboral Hondureño, 2013. Perspectivas. 1(1). 38-49.

Recibido:

Noviembre 15 de 2015

Aceptado:

Febrero 05 de 2016

\section{Resumen}

En el presente artículo se aborda el tema de la participación laboral de la mujer en Honduras, donde para el año 2013 la mujer representó un 52.8\% de la Población en Edad de Trabajar (PET) y un 36.5\% de la Población Económicamente Activa (PEA). En el artículo se indica la importancia de la inserción femenina en el mercado laboral, debido a ello se realiza un análisis de los factores que inciden en su participación. El objetivo principal del estudio es determinar la incidencia de variables relevantes para la inserción de la mujer dentro de la Población Económicamente Activa (PEA). Además se realiza un análisis descriptivo de la situación de la mujer en el mercado laboral hondureño. La investigación es de corte cuantitativo y por ende se realiza un modelo de regresión lineal para determinar la probabilidad de inserción de la mujer en el mercado laboral. El modelo determinó que entre los factores que impactan en forma negativa: es el estado conyugal, si la fémina es pobre y si recibe remesas familiares; mientras que, la edad, el nivel educativo alcanzado, la rama de activa en que se desempeña y si es jefe de hogar guardan una relación positiva en la probabilidad que se inserte en las filas de la población activa, no obstante, la zona o dominio donde reside y si recibe transferencias del gobiernos mostraron ser poco significativas dentro del estudio.

Palabras claves: Inserción laboral de la mujer, población económicamente activa, población en edad de trabajar.

\section{Abstract}

This article presents the topic of the labor participation of women in Honduras; in 2013, women represented $52.8 \%$ of the working-age population and $36.5 \%$ of the economically active population (EAP). The article indicates the importance of female insertion in

\footnotetext{
*Esta investigación parte de estudios previos del mercado laboral en Honduras como parte de trabajos de tesis, artículos de revistar y presentación en congresos (Congresos de Economía Administración y Tecnología (CEAT) y XXX Congreso ALAS).

**Licenciada en Economía, Oficial de Investigación en el Instituto de Investigaciones Económicas y Sociales (IIES) de la Universidad Nacional Autónoma de Honduras (UNAH), kmeza@iies-unah.org. TEL+50422391849.

*** Licenciado en Economía, Oficial de Investigación en el Instituto de Investigaciones Económicas y Sociales (IIES) de la Universidad Nacional
Autónoma de Honduras (UNAH). jcabrera@iies-unah.org

****Licenciado en Economía y pasante de la carrera de matemáticas, voluntario en el Instituto de Investigaciones Económicas y Sociales (IIES) de la Universidad Nacional Autónoma de Honduras (UNAH).mramos@unah.hn
} 
the labor market, that's why an analysis of the factors that affect their participation is carried out. The main objective of the study is to determine the incidence of relevant variables in the insertion of women within the Economically Active Population (EAP). In addition, a descriptive analysis of the situation of women in the Honduran labor market is carried out. The research is a quantitative one; a linear regression model is performed to determine the probability of insertion of women in the labor market.

The model determined that among the negatively impacting factors are marital status, if the female is poor and if she receives family remittances; While, the age, the educational level, the branch of activity in which she performs and if she is a head of household keep a positive relation in the possible insertion in the lines of the active population, nevertheless, the zone or domain where she resides and if she receives transfers of the government showed little significance in the study.

Key words: Employment of women, economically active population, working age population.

\section{Resumo}

Neste artigo, é abordada a questão da participação do trabalho das mulheres em Honduras, onde em 2013 as mulheres representavam o 52,8\% da população em idade activa e um $36,5 \%$ da população economicamente ativa. No artigo destaca-se a importância das mulheres que entram no mercado de trabalho, desenvolvendose uma análise dois fatores que afetam na sua participação. O objetivo principal do estudo é determinar a incidência de variáveis relevantes para inserção da mulher na população economicamente activa. Também e apresentada uma análise descritiva da situação das mulheres no mercado de trabalhona Honduras .A pesquisa teve caráter quantitativa e portanto é feito um modelo de regressão linear para determinar a probabilidade de inclusão da mulher no mercado de trabalho. $\mathrm{O}$ modelo revelou que entre os fatores que impactam negativamente encontram-se: estado civil, se a fêmea é pobre e se recebe remessas familiares; enquanto que,a idade, o nível de instrução, o ramo de atividade que executa, se for chefe de familia é positivamente relacionada com a probabilidade de que seja inserida nas filas da população activa, no entanto, a zona ou domínio onde reside e recebe transferencias do governo mostraram ser insignificantes no estudo.

Palavras chave: inserção laboral da mulher, população em idade activa, população economicamente activa.

\section{Introducción}

La participación de la mujer en el mercado laboral ha estado cambiando significativamente en la última década, siendo esto un aporte positivo en el desarrollo de un país. Anteriormente la mujer sólo era considerada como parte fundamental de los quehaceres del hogar y la crianza de los hijos, sin embargo, en la actualidad puede estar involucrada en ambos aspectos de su vida: familia y trabajo.
La economía latinoamericana en el año 2014 no generó los suficientes empleos, mostrando una baja tasa de participación 
Vol. 1

No. 1

Jul - Dic 2016

ISSN: 2590-9215

(En línea)

PP: $38-49$ reducir la pobreza esto debido al nuevo rol de la mujer en el mercado laboral en la última década, con un mayor empoderamiento y con superiores niveles educativos (Organización Internacional del Trabajo, 2014, p. 36) .

El Informe sobre Desarrollo Humano para Honduras 2008/2009 hizo referencia a la exclusión social juvenil -que se entiende como la privacidad de las capacidades de los jóvenes en su vida económica y social. Este informe destacó que la educación es un factor significativo para ir menguando la exclusión social, ya que está influye en el momento de obtener un mejor empleo, además mostró que la mujer tiene mayor nivel educativo que el hombre.

No obstante, la mujer fue la población más desempleada y con menores salarios, sin poder contabilizar su aporte a la actividad económica (por los trabajos que realiza también en el hogar), donde la problemática radica que los empleadores mira la población femenina como un potencial para ser madres de familia lo que implica costos de maternidad, según los beneficios que establece la ley laboral (artículo 135 del código de trabajo) y aun así, son las que más cotizan en la seguridad social; esto influencia a la rama de actividad que se ubican al sector secundario y terciario de la economía (industria manufacturera y docencia) (Programa de las Naciones Unidas para el Desarrollo (PNUD) , 2009, pp. 30-37).

Debido informes de la OIT laborales, Banco 40 Mundial y PNUD que indican la importancia de la mujer en el mercado laboral, por ello es necesario conocer ¿cuáles son los factores que inciden en la participación de la mujer en el mercado laboral de Honduras?, considerando variables como: edad, dominio, estado civil, rama de actividad, si recibe remesas familiares, si es jefe de familia, años de escolaridad y pobreza.

Debido a ello, este artículo ha realizado una revisión de investigaciones relacionadas con la participación laboral de la mujer, teorías referentes al mercado laboral, incluyendo un análisis descriptivo de las variables consideradas significativas según la lectura realizada en la investigación y finalmente la verificación de la participación bajo un modelo de regresión.

\subsection{Antecedentes}

Moráis (2012) en su tesis realizó un modelo probit y logit para establecer cuáles son los principales factores que influyen para que una mujer participe en el mercado laboral. En esta indico que es necesario conocer su contexto familiar, para determinar donde implementa su tiempo disponible. Mostrando que la mujer será parte de la Población Económicamente Activa (PEA) ${ }^{1}$ siempre que su tasa de salario real exceda la productividad marginal del trabajo doméstico y su salario doméstico.

Además que la población femenina se ubica en el sector terciario (docencia, salud, comercio) de la economía del Amazonas por la segmentación laboral existente entre las mujeres y los hombres que ellas tienen a ubicarse en el sector de servicios debido a los trabajos realizados en dicho sector y en la zona urbana tenían mejor salarios que la zona rural.

Las variables estado civil y si existen hijos en edad preescolar fueron significativas en la zona urbana; esto debido que en la área rural envían sus hijos al campo a trabajar, también indicando que si existe, otro adulto que no trabaja, ni estudia; existe más probabilidad que la mujer se inserte en mercado laboral. Moráis considera la implementación de una política para qué la mujer al momento de casarse no reduzca su inserción en mercado laboral.

${ }^{1}$ La PEA son todas las personas de 10 años (en Honduras) y más que tienen empleo o que no teniéndolo, están buscando o se encuentran a la espera de alguno. 
Méndez (2010) resaltó que en Chile en los últimos 10 años se han dado cambios en el mercado laboral, por ello era necesario conocer cuáles factores habían influido, con una estimación de corte transversal para los años 1996, 2001 y 2006, donde se evidenció que las variables que tienen una relación negativa para que una mujer se inserte en mercado laboral son: ingreso per cápita del núcleo familiar, presencia de un cónyuge, la presencia de hijos (en edad preescolar) y la contracción de la economía y los factores positivos: que exista otro adulto en el hogar que no trabaje, ni estudie, el nivel educativo, que esté en la zona urbana y una mayor actividad económica.

Concerniente al etario de la mujer de 15-19 años y de 45 en adelante se le dificulta más ser parte de la Población Económicamente Activa (PEA) y los hombres de 25-44 años. Revelando que la segmentación laboral es distinta, la mujer se dedica al comercio, servicios comunales y agricultura, y el hombre a la minería, construcción y servicios financieros.

No obstante, en la remuneración salarial por hora el hombre gana $28.6 \%$ más que la mujer y finalmente que la mujer soltera sin hijo o soltera con hijos tiene una mayor participación en el mercado laboral y la mujer no soltera $45 \%$ menos de probabilidad, aunque esto dependía del nivel educativo alcanzando. Manifestando que la mujer toma decisiones con relación a la maternidad, por lo que, si no existe horarios flexibles y licencias de maternidad o una persona mayor que cuide a su hijo, dejará de ser parte de la población ocupada

Por otra parte, Contreras y Plaza (2007) indicó que no solo las variables: edad, estado civil, escolaridad y número de hijos, sino que las características culturales (el machismo $\mathrm{y}$ valores) las que afecta negativamente la participación de la mujer en el mercado laboral. Tal afirmación se sustenta en la encuesta International Social Survey Program (ISSP) realizada en el año 2002, que mostro una relación inversa entre el nivel educativo y el machismo.

Destacan que los hombres separan su vida laboral de los trabajos domésticos y la crianza de los hijos; considerando que esos roles son solamente de la mujer. En un modelo simple de decisiones un individuo busca maximizar su nivel de bienestar dependiendo de su consumo y su ocio. Sin embargo, sacrificara su ocio por trabajo, donde recibe ingresos para el consumo, aunque se presentan restricciones en el caso de la mujer tales como: ingreso laboral, número de hijos y restricciones sociales. Por ello es necesario menguar el machismo en la población.

Aunque la mujer se está insertando en el mercado laboral lo ha realizado en las ramas de actividad con menos crecimiento (servicios y comercio). Señalando que el mayor número de desempleadas son la de educación superior, esto podría están surgiendo por empleos menos especializados generados en el país.

Con 4 modelos logit se determinó que las variables que influyen positivamente en la inserción laboral son: educación, estado civil y jefatura del hogar. En contrasté, incide negativamente la tenencia de hijos entre 0-6 años. Finalmente, la edad y situación conyugal no fueron significativas en el caso de Cartagena según Hernández (2011).

En los antecedentes se encontró que la población femenina ha sido más afectada por el desempleo en los países de Latinoamérica (Chile, Brasil Colombia y entre otros), aun así la población ocupada enfrenta otra problemática la informalidad en el mercado laboral donde no se generan empleo formales se es incapaz de obtener: contratos laborales, acceder a seguridad social es decir que la mujeres están expuestas a no contar con cuidados prenatal y posnatal dificultando en
No. 1

Jul - Dic 2016

ISSN: $2590-9215$

(En línea)

PP: $38-49$ 
ocasiones su reintegro al mercado laboral.

Asimismo se logró identificar otros factores como: edad, estado civil, si tiene hijo en edad preescolar, actividad económica, nivel educativo, características culturales, jefatura del hogar y dominio.

\subsection{Teoría de Capital Humano}

Organización para la Cooperación y el Desarrollo Económicos (2007) define Capital Humano como la mezcla de aptitudes y habilidades propias de las personas; y la calificación y el aprendizaje que adquiere en la educación y la capacitación (p.2).

Becker (1977) considera el creciente del trabajo como Capital Humano, que es la inversión en las personas (en educación y capacitaciones) para elevar y conservar la productividad y mejorar los rendimientos laborales; esto debido que él Capital Humano se valora como un activo que promueve el crecimiento y la empleabilidad.

De esta manera, se enfoca en responder tres preguntas de Capital Humano: i. ¿Que determina el número de seres humanos? mencionando que el crecimiento de la población está determinada por las tasas de natalidadymortalidad; ademásqueenlospaíses desarrollados el crecimiento de la población no está establecida por necesidad fisiológica, sino por una variable socioeconómica es decir que el tamaño de la familia está determinado por su nivel de ingresos y que costo del tiempo de la madre es de suma importancia, pero esto dependerá del valor que tenga para ella, lo cual restringirá el número de hijos; ii. ¿Que determina la participación en la fuerza laboral? las personas también determinan sus horas de trabajo disponible, puede estar realizado actividades con pagos monetarios (actividades de mercado) o actividades de que rinden satisfacción (están fuera del mercado y de consumo) y el tiempo que no se trabaja se llama ocio excluyendo actividades como: cuidado de los niños, limpieza del hogar y dormir, en la actividades fuera del mercado la mujer tiende a trabajar menos que sus esposos y ellas dedican más tiempo a las actividades domésticas por ende su tiempo es más barato, sin embargo al aumentar su tiempo en el mercado reduce su tiempo en hogar; iii. ¿Que determina la selección ocupacional? Esto dependerá de la habilidad de la personas y el tiempo dedicado al trabajo, igualmente se dividirá entre distintas ocupaciones dependiendo su grado de especialización donde su fuente de beneficio es la educación y las capacitaciones que aumentan la productividad de un ocupación (pp. 2012018).

Según Schultz (1961) la adquisición de conocimiento y habilidades de las personas es una parte importante de la riqueza de una nación, indicando que el trabajo es único. Con la inversión en las personas se puede mejorar sus opciones de elección dejando a los individuos un camino libre para mejorar su bienestar, eso debido a que los recursos humanos tienen dos dimensiones: cuantitativas y cualitativas; el número de personas y horas trabajadas son características medibles, sin descuidar los componentes cuantitativos como el conocimiento y las habilidades en la medida que se incrementa la inversión en ellas, incrementara el valor productivo de la fuerza laboral rindiendo una tasa de retorno positiva.

Para mejorar las capacidades humanas un país debe concentrarse en cinco categorías: 1. Facilidad en la salud y sus servicios para mejora la esperanza de vida, 2. La formación del trabajo donde los hombres y mujeres con menos edad se mueven a mejores oportunidades laborales dependiendo del nivel educativo alcanzado, 3. Educación organizacional formal el logro de nivel educativos superior es un factor clave, siendo más significativo en el caso de las mujeres, 4. Programas para adultos debido a que 
la inactividad afecta ya que se deprecia la adquisición de habilidades y conocimiento y 5. La migración de los individuos y familias para ajustarse a los cambios de oportunidades de empleo (pp. 5-9).

\section{Metodología}

La investigación es de enfoque cuantitativo con alcance descriptivo-exploratorio, debido que intenta encontrar los factores que inciden para que la mujer hondureña participe en el mercado laboral y además, se establece los componentes más significativos en el mercado laboral para las mujeres. También la investigación es de tipo exploratorio, ya que hace una relación entre las variable dependiente con las independientes, con un modelo de regresión lineal que colabora a determinar cuál es la probabilidad de que una mujer participe en mercado laboral.

Al ser pobreza una de las variables consideradas dentro de la investigación, se apega metodológicamente a la definición que establece el Instituto Nacional de Estadísticas de Honduras "Consiste en establecer, a partir de los ingresos de los hogares, la capacidad que estos tienen para satisfacer, por medio de la compra de bienes y servicios, un conjunto de necesidades alimentarias y no alimentarias consideradas como básicas" (INE, 2015), si bien existen muchas definiciones de pobreza, el método que se utiliza en el estudio es el que establece el INE, por lo cual al establecer pobreza será el concepto previamente establecido.

Para la recolección de las variables se utilizó datos de la Encuesta Permanente de Hogares de Propósitos de Múltiples (EPHPM) del año 2013 elaborada por Instituto Nacional de Estadísticas (INE), y tomando en cuenta las variables que según la literatura encontrada pueden ser relevantes para el estudio, por lo cual previo a la elaboración del modelo fue necesario filtrar las variables, obviando todas las que no fueron necesarias.
Es importante mencionar que una de las limitaciones encontradas ha sido la disponibilidad de la encuesta EPHM en sus publicaciones de los años 2014 y 2015, en primera instancia la del año 2015 debido a que no se había terminado de realizar cuando se estaba realizando el presente estudio, y la del año 2014 debido a la política de restricción de uso público que posee el INE.

\subsection{Especificación del Modelo}

La estimación econométrica realizada es un modelo de regresión lineal simple en el cual se utilizan variables sociales las cuales son dicotómicas o binarias (toman valores de $0 \mathrm{y}$ 1) dado la naturaleza de la investigación se tomaron las variables que según se establece en secciones anteriores determinan la probabilidad de que una mujer se inserte a la PEA, es de destacar y aclarar que el modelo trata de explicar lo probabilidad de que una mujer esté dentro de las personas activas que poseen empleo o lo andan buscando, y no directamente es explicar la probabilidad de que una mujer posea empleo o no, además es de resaltar que la estimación se realizó en el software STATA12.

Forma funcional:

Dónde:

PEAfem: Son las mujeres que están activas en el mercado laboral (empleadas o desempleadas)

Cónyuge: representa las mujeres que tienen presencia de un cónyuge sean casadas o de unión libre.

Jhogar: representa las mujeres que son jefas de hogar.

Pobref: representa las mujeres que se encuentran en pobreza.

Educ: representa los años de estudio que posee.

Dom1: representa la zona de residencia en este caso la zona urbana.
No. 1

Jul - Dic 2016

ISSN: 2590-9215

(En línea)

PP: $38-49$ 
ramaagric: representa las mujeres que están en la rama de actividad económica de agricultura.

ramaindust: representa las mujeres que están en la rama de actividad económica de industria.

ramaelec: representa las mujeres que están en la rama de actividad económica de electricidad.

ramacom: representa las mujeres que están en la rama de actividad económica del comercio.

ramatrans: representa las mujeres que están en la rama de actividad económica de transporte.

ramafinac: representa las mujeres que están en la rama de actividad económica de servicios financieros.

Ramaserv: representa las mujeres que están en la rama de actividad económica de servicios edadpfem: representa la edad de las mujeres.

B10mil: representa si las mujeres reciben el bono 10 mil.

Remesas: representa si la mujer recibe remesas del exterior.

\section{Resultados}

\subsection{Análisis Descriptivo del Panorama Laboral de la Mujer en Honduras}

Con la crisis internacional en el 2009, el salario del hombre se vio fuertemente contraído. Se destaca en esta crisis que la participación de la mujer ayudó a que el impacto no fuera más considerable y que la pobreza no aumentará. Sin embargo, la segmentación laboral, la desigualdad en los salarios y las agencias (donde ella sea tomadora de decisiones) no han ayudado a una mayor inserción.

Cabe destacar, que la mujer en una década ha ayudado a reducir la pobreza en $30 \%$, el hombre un $39 \%$ y el resto otros factores (remesas familiares, programas de transferencias, etc.); siendo vulnerables los hogares que solo dependen de un solo ingreso laboral y que la mujer es jefe de hogar.

En el caso de Honduras la tasa de participación de la mujer fue negativa de $-3 \%$ del 2000 2010 en el rango etario de 15-34 años. En Latinoamérica, la mujer de bajos recursos es la que más se ha insertado en mercado laboral en relación a las mujeres ricas (Panamá, Costa Rica, Perú), siendo caso contrario en Honduras que la partición se redujo en 12\% (Banco Mundial , 2012).

En Honduras, la mujer representó para el 2013 un 52.8\% de la Población en Edad de Trabajar (PET) con un dominio en área urbana de $60.1 \%$, no obstante, de la Población Económicamente Activa (PEA) sólo representó un 36.5\% y una Tasa de Participación (TP) de 37.2\%. Debido a la reducida participación de la mujer en el mercado laboral en el gráfico $\mathrm{N}^{\circ} 1$, se muestran las variables que según la lectura realizada en la investigación son relevantes para que una mujer se inserte en el mercado trabajo.

Para el 2013 de la Población Económicamente Activa femenina un $43.56 \%$ contaba con un cónyuge en el hogar; un $44.57 \%$ de ellas tenían educación primaria, 32.69\% educación secundaria y solo un $12.87 \%$ educación superior; en las ramas de actividad que más participan son: comercio (38.2\%), Servicios Comunales, Sociales y Personales (27.31\%) e Industria Manufacturera (18.3\%); en relación al etario de edad de 10-35 años representaron un $51.35 \%$ de la Población Económicamente Activa femenina; un $84 \%$ de ellas indicó no recibir ninguna transferencia del gobierno y solo un $6.28 \%$ reciben remesas familiares.

El factor más preocupante es que un $25.58 \%$ de las mujeres exteriorizaron ser jefas de hogar considerándose un efecto negativo para reducir la pobreza y finalmente de la Población Económicamente Activa femenina un $54.7 \%$ son pobres.

Se puede deducir que la población femenina se ha visto más afectada por el desempleo aunque en la actualidad tenga mayores años de 
estudio en el país. Con este escenario podría repercutir negativamente en el largo plazo en la estructura familiar y con ello desmejorar los indicadores económicos esto debido a la importancia de la mujer para reducir la pobreza y su influencia en la educación de los infantes.

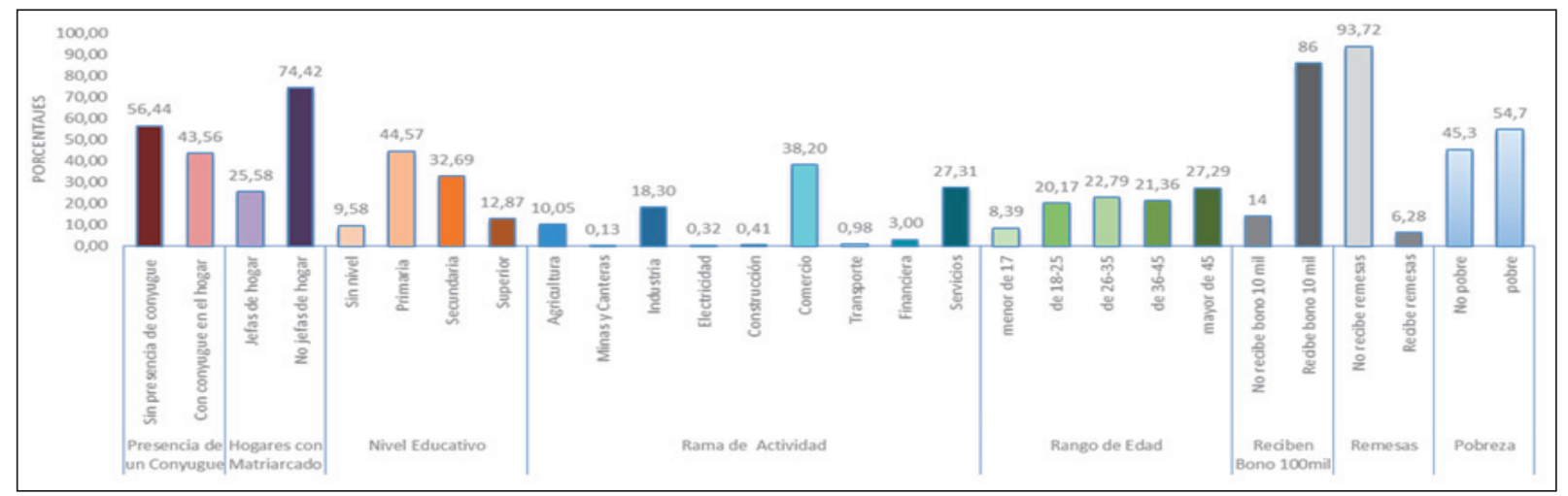

Figura $N^{\circ}$. Participación de la Mujer en Mercado Laboral en Honduras año 2013. Fuente: Elaboración propia con datos de la Encuesta Permanentes de Hogares con Propósitos Múltiples

\subsection{Resultados de la regresión lineal}

Con datos de Encuesta de Permanente de Hogares de Propósitos de Múltiples (EPHPM), se realizó un modelo de corte transversal para determinar la probabilidad que una mujer se inserte en el mercado laboral, según las variables descritas anteriormente y verificadas según la teoría se realizó un análisis de los coeficientes obtenidos. El modelo resultó significativo con una bondad de ajuste de 0.8426 con los siguientes resultados (ver figura $\mathrm{N}^{\circ}$ 2).

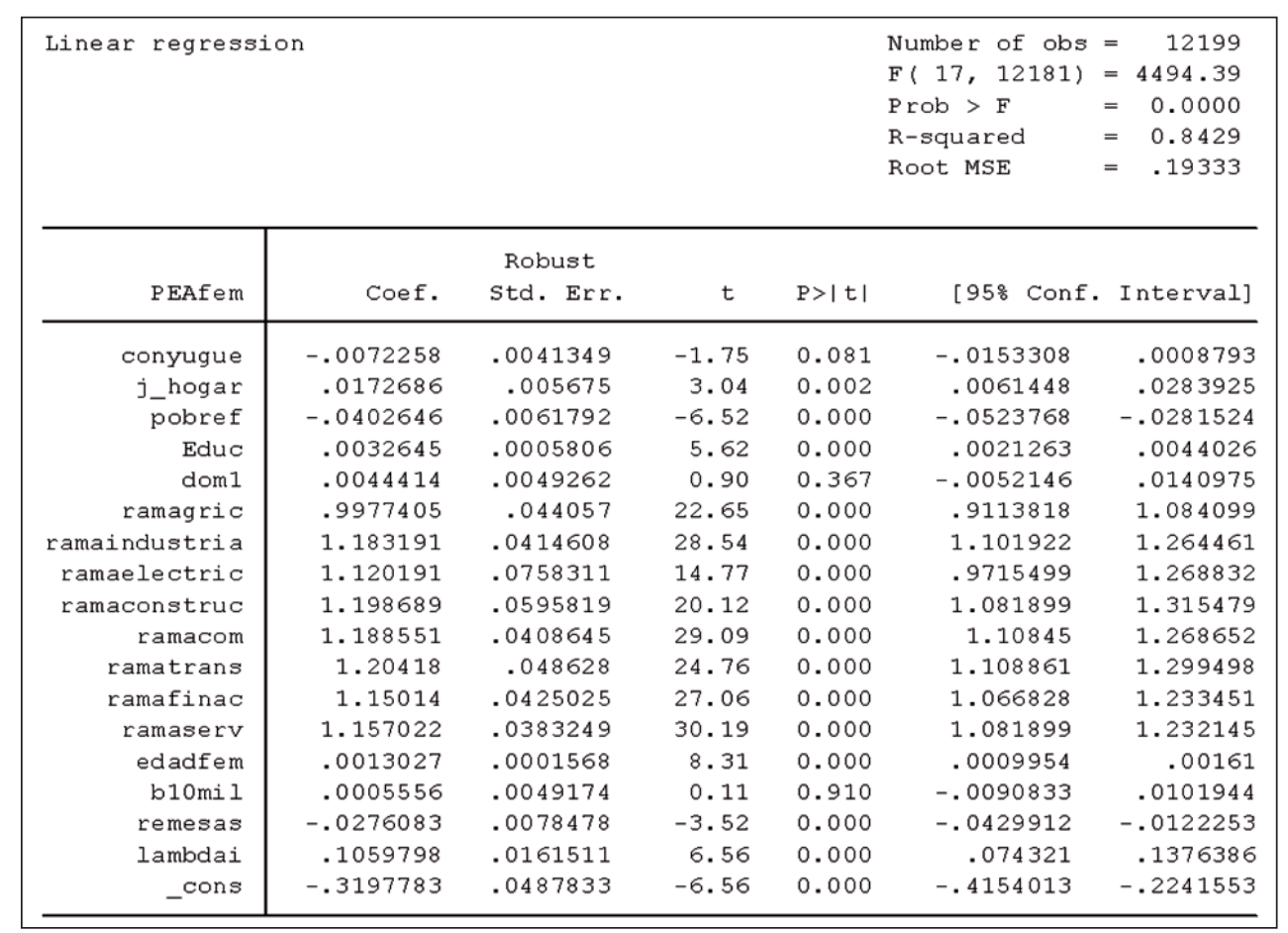

Figura $N^{\circ} 2$ Regresión Lineal de la probabilidad de inserción de la mujer hondureña a la Población en Edad de Trabajar (PEA). Fuente: Elaboración propia con datos de EPHPM 
Vol. 1

No. 1

Jul - Dic 2016

ISSN: $2590-9215$ (En línea)

PP: $38-49$
De acuerdo a los resultados expuestos en la regresión se puede hacer las siguientes acotaciones:

i. La mujer que cuenta con un cónyuge ya sea casada o en unión libre disminuye su probabilidad de insertarse en el mercado laboral en un $0.72 \%$. Resaltando que este resultado es similar a otras investigaciones de países de América latina.

ii. No obstante, para las mujeres que se desempeñan como jefes de hogar la relación es distinta a las que cuentan con un cónyuge, mostrando una probabilidad de participación de $1.7 \%$;

iii. En cuanto a las mujeres que se encuentran en condición de pobreza, tienen un $4 \%$ menos de probabilidad de ser parte de la Población en Edad de Trabajar, cabe resaltar que el Banco Mundial menciona que la población femenina que se encuentra en pobreza es la que menos se inserta en el mercado laboral en comparación con la de ingresos medios y altos,

iv. El nivel educativo que logre una mujer mostró ser significativo, es decir, que por cada año más de educación aumenta en $0.326 \%$ la probabilidad de insertarse en mercado laboral. Siendo uno de los factores con mayor grado de significancia para una mayor participación.

v. Si las mujeres residen en la zona urbana, aumenta su probabilidad en $0.044 \%$ de ser parte activa del mercado laboral, aunque es de resaltar que es una variable poco significativa en el modelo.

vi. Todas las ramas de actividades económicas mostraron ser significativas, es decir que las mujeres podrían insertarse en cualquiera de ellas, sin embargo, es de resaltar que la rama de agricultura es en la cual las mujeres tienen menos probabilidad de insertarse en relación a las demás.

vii. A las mujeres de menos edad le resulta más difícil insertarse en el mercado laboral. En este estudio se demuestra que para el caso de Honduras que por cada año más de vida su probabilidad de inserción aumenta en $0.13 \%$.

viii. El bono 10 mil es un incentivo que el gobierno de la República de Honduras brinda a las madres con el fin de que tengan a sus hijos en los centros educativos, el resultado de este coeficiente resultó ser no significativo $(0.0056 \%)$ esto debido que no es una gran población que lo recibe y además que el incentivo monetario es poco (aproximadamente 435\$US al año) como para que una mujer deje de buscar empleo.

ix. El coeficiente de remesas familiares indicó que la mujeres que perciben dinero del exterior disminuyen su probabilidad en $2.7 \%$ de insertarse en el mercado laboral.

Es importante mencionar que la investigación no se orienta a explicar las causas o la correlación entre variables cualitativas y las variables consideradas dentro del modelo, sino que se limita a encontrar el porcentaje de incidencia de variables específicas en relación a la probabilidad de entrar al mercado laboral, por lo cual será necesario realizar otro estudio que permita profundizar en variables importantes de corte cualitativo, como ser la desintegración familiar, igualdad de género, el rol de la madre en el hogar y cuidado de los hijos, entre otras.

\section{Discusión de resultados}

García y Cortes (2012) concluyen que el progreso de la mujer en el mercado laboral no se ha desarrollado de manera uniforme y dependiendo de la edad, educación de ella y las características familiares. Otro resultado es que la mujer reduce su probabilidad de participación si sus ingresos difieren de los otros miembros de la familia (p. 44). Esto relacionado con la teoría de capital humano donde Becker menciona que la participación de la mujer depende del valor que tenga para 
ella. Mencionado lo anterior, es de resaltar que resultados similares se encontraron en esta investigación, si bien no profundiza en encontrar la relación de los ingresos del conyugue tal como lo hace García y Cortes, tiene similitud a lo encontrado por estos autores principalmente en la reducción de la probabilidad de la participación laboral de la mujer en presencia de un conyugue. Otro importante aspecto es la edad, para el caso de Honduras se determina que la mujer entre más joven sea menos probabilidad de insertarse en un trabajo tiene, lo indicado por García y Cortes es afín a los resultados encontrados en esta investigación.

En la teoría de Capital Humano Schultz resalta la importancia que las personas adquieran conocimiento y habilidades que da como resultado una mejora en su bienestar, en la segunda categoría de capacidades humanas se alude sobre la formación en el trabajo donde la población joven con mayor capacidades puede mover en distinto trabajos con más facilidad y el tercero de educación organizacional formal la mujer es más beneficiada al lograr un nivel educativo superior. Esto se ve reflejado en las conclusiones de Ferrada y Zarzosa (2010) donde la mujer casada tiene menos probabilidades de insertarse en el mercado laboral, sin embargo, si la mujer casada tiene educación superior aumenta su participación según las regiones analizadas y otro resultado es la población femenina joven tiene menos probabilidades de participación (p. 97). En el caso de Honduras se refleja un comportamiento similar en dichas variables donde la mujer con nivel superior tiene mayor participación y menos cuando es joven una tendencia similar a los países de América Latina que sirvieron de apoyo en la bibliografía.

En alusión a los demás resultados del modelo realizado, es importante resaltar que las mujeres hondureñas pueden insertarse en cualquier rama de actividad económica, a excepción de la rama de agricultura, la razón de ello es que este trabajo se da en la zona rural y es un trabajo eminentemente físico, en cuanto a la rama de actividad donde la mujer se inserta con mayor facilidad es el área de servicios, derivado de que en esta rama de actividad se exige un nivel educativo un tanto mayor que en las demás, el cual la mujer adquiere dado que obtiene un mayor bienestar $\mathrm{y}$ un mejor ingreso en esa rama de actividad, tal como menciona Becker que la mujer se insertará en el mercado laboral siempre y cuando los beneficios compensen el tiempo que dejaran de estar con sus hijos.

\section{Conclusiones}

La mujer como parte del mercado laboral es un factor clave para la reducción de pobreza, sin embargo, existe factores como se resalta en la investigación que obstaculizan su inserción y la permanencia de ellas en dicho mercado. Un aspecto importante a mencionar es la presencia de un conyugue, ya que cuando esto ocurre las mujeres reducen su probabilidad en $0.72 \%$ de pertenecer a la Población Económicamente Activa, si bien esto afecta en este sentido, no se puede dejar de lado los beneficios al hogar que la presencia de ambos padres. En contraste a lo anterior mencionado, cuando la mujer es jefa de hogar, la necesidad de empleo aumenta y trae consigo un incremento de $1.7 \%$ de probabilidad de insertarse en la PEA que de las mujeres que nos son jefes de hogar.

Las mujeres en situación de pobreza, también mengua la probabilidad de participación de la ellas en el mercado laboral; en un 4\%. Esta relación es debido a la dificultad de poder acceder a educación formal, disminuyendo con esto su posibilidad de poder salir de la pobreza y optar a mejores trabajos, tal como lo menciona el Banco Mundial (2012).

Dado que las remesas que mandan las personas que laboran en el exterior del país son un sustento importante para las familias, las féminas hondureñas se ven
No. 1

Jul - Dic 2016

ISSN: 2590-9215

(En línea)

PP: $38-49$ 
menos incentivadas a insertarse dentro del mercado laboral por lo cual el resultado para esta variable fue una disminución de $2.7 \%$ de probabilidad cuando una mujer recibe remesas.

Apegándonos a lo mencionado por Becker resulta interesante como los resultados de la investigación guardan una estrecha relación con la teoría del capital humano, teniendo en cuenta que esta menciona que la educación o el aprendizaje especializado son el principal motivo de empleabilidad y productividad en una nación, por lo cual el resultado de la investigación denota una evidencia a lo mencionado por Becker, para el caso de Honduras la educación es un aspecto importante y de alta significancia para que una mujer decida insertarse en el mercado laboral, dando como resultado que cada año adicional de educación en una mujer, aportará un incremento de $0.326 \%$ a la probabilidad de estar dentro de la Población Económicamente Activa.

\section{Bibliografía}

Becker G. (1977). Teoría de Capital Humano. Teoría Económica (1 a ed, pág. 139150). México: fondo de cultura económica.

Contreras D. y Plaza G. (2007). Participación laboral femenina en chile. ¿Cuánto importan los factores culturales? Recuperado el 10 de octubre de 2015 de http://www.econ.uchile.cl/uploads/ publicacion/41f86d8f-4439-4b90 862d-0e3c78bb85f3.pdf.

García J. y Cortez P. (2012). Análisis de la participación laboral de la mujer en el mercado ecuatoriano. Recuperado de https://dialnet.unirioja.es/ ejemplar/361782.

Hernández N. (2001).Determinantes de la participación femenina en el mercado laboral: caso Cartagena.
Recuperado el 15 de septiembre de 2015 de http://190.25.234.130:8080/ jspui/bitstream/11227/422/1/ D E T E R M I N A N T E S \% 20 $\begin{array}{llllllllll}\mathrm{D} & \mathrm{E} & \% & 2 & 0 & \mathrm{~L} & \mathrm{~A} & \% & 2 & 0\end{array}$ PART ICIPACI\%C 3\%93 N\% 20 FEMENINA \%20EN\%20EL\%20 MERCADO\%20LAB.pdf.

Instituto Nacional de Estadísticas (2013). Encuesta Permanente de Hogares de Propósitos Múltiples.

Instituto Nacional de Estadísticas (2015). Método de la línea de pobreza. Recuperado el 2 de enero de 2016 de http://www.ine.gob.hn/index.php/ component $/$ content/article?id $=91$

Morais G. (2012). Determinantes da participação feminina no mercado de trabalho amazonense. Recuperado el 10 de septiembre de 2015 http:// alexandria.cpd.ufv.br:8000/teses/ economia/2012/242962f.pdf.

Méndez C. (2010). Los determinantes de la participación laboral femenina. Chile: 1996, 2001 y 2006. Recuperado el 28 de agosto de 2015 de http://repositorio. uchile.cl/tesis/uchile/2010/cfmendez_cv/pdfAmont/cf-mendez cv.pdf

Organización de Internacional del Trabajo (2014). Panorama laboral 2014 América Latina y el Caribe. Recuperado el 15 de noviembre de 2015 de http://www.ilo.org/ wcmsp5/groups/public/---americas/--ro-lima/documents/publication/ wcms_325664.pdf.

Organización para la Cooperación y el Desarrollo Económicos (2007). Capital humano: Cómo moldea tu vida lo que sabes. Recuperado de https:// www.oecd.org/insights/38435951.pdf 
Programa de las Naciones Unidas para el Desarrollo (2009).Informe sobre Desarrollo Humano Honduras 2008/2009. Recuperado el 25 de octubre de 2015 de http://www. hn.undp.org/content/dam/honduras/ docs/publicaciones/HN_PNUD2009 IDH.pdf.

Shultz T (1961). Investment in Human Capital. Recuperado el 5 de octubre de 2015 de http:/www.ssc.wisc.edu/ walker/ wp/wp-content/uploads/2012/04/ schultz61.pdf

World Bank (2012). The effect of women's economic power in Latin America and the Caribbean. Recuperado el 15 de agosto de 2016 de http:// www.bancomundial.org/content/ $\mathrm{d}$ a m / Wor ld bank/d o cument / PLBSummer12latest.pdf 